PAPER • OPEN ACCESS

In situ scanning x-ray diffraction reveals strain variations in electrochemically grown nanowires

To cite this article: Alfred Larsson et al 2021 J. Phys. D: Appl. Phys. 54235301

View the article online for updates and enhancements.

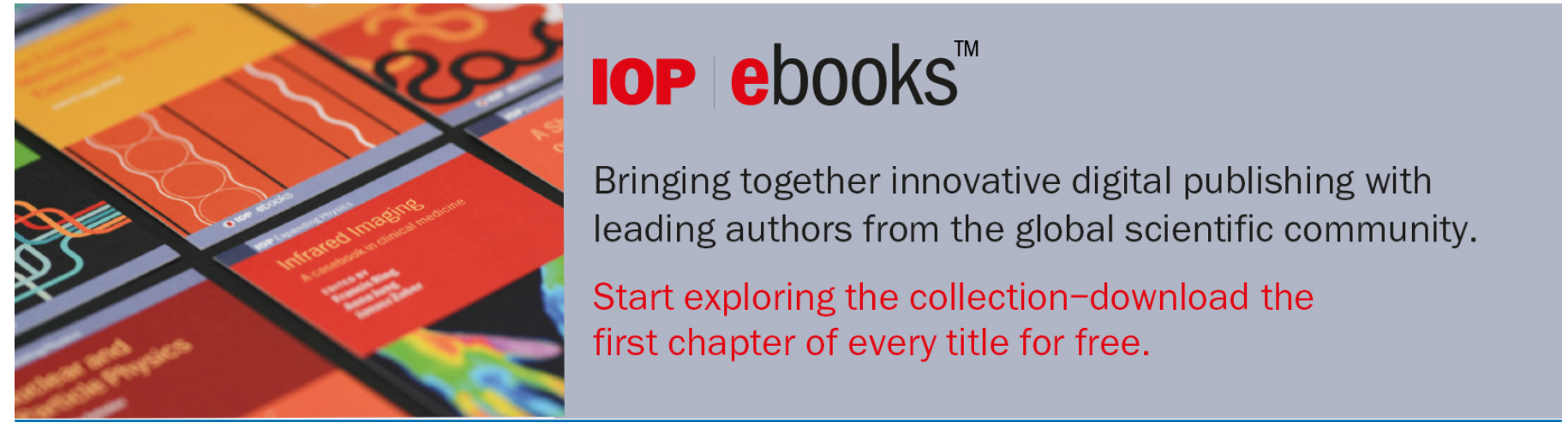




\title{
In situ scanning $x$-ray diffraction reveals strain variations in electrochemically grown nanowires
}

\author{
Alfred Larsson ${ }^{1,2, *}\left(\mathbb{D}\right.$, Giuseppe Abbondanza ${ }^{1,2}(\mathbb{D})$, Lisa Rämisch ${ }^{3}$, Weronica Linpé ${ }^{1,2}$, \\ Dmitri V Novikov ${ }^{4}$, Edvin Lundgren ${ }^{1,2}$ (D) and Gary S Harlow ${ }^{1,2,5, *}$ (D)
}

${ }^{1}$ Division of Synchrotron Radiation Research, Lund University, Lund, Sweden

${ }^{2}$ NanoLund, Lund University, Box 118, 22100 Lund, Sweden

${ }^{3}$ Division of Combustion Physics, Lund University, Lund, Sweden

${ }^{4}$ DESY Photon Science, Hamburg, Germany

${ }^{5}$ Department of Chemistry, Nano-Science Center, University of Copenhagen, Universitetsparken 5,

DK-2100 Copenhagen $\varnothing$, Denmark

E-mail: alfred.larsson@sljus.lu.se and gary.harlow@chem.ku.dk

Received 9 December 2020, revised 2 February 2021

Accepted for publication 2 March 2021

Published 16 March 2021

\begin{abstract}
Templated electrochemical growth in nanoporous alumina can be used to fabricate nanowires with applications in magnetic storage devices, hydrogen sensors, and electrocatalysis. It is known that nanowires, grown in such templates, are strained. The strain in nanoscale materials can influence their performance in applications such as catalysts and electronic devices.

However, it is not well established how the nanoporous template affects the lattice strain in the nanowires and how this develops during the growth process due to the lack of non-destructive in situ studies with spatial resolution. We have measured the strain and grain size of palladium nanowires in nanoporous templates during the growth process. For this, we performed in situ scanning x-ray diffraction with a submicron focused $\mathrm{x}$-ray beam. We found a tensile strain in the nanowires and that it is more pronounced along the growth direction than in the confined direction of the templates. The tensile strain measured in situ is higher than previous ex situ reports, possibly due to hydrogen absorption during the growth. With the spatial information made possible with the focused synchrotron x-ray beam, we could observe local variations in the strain as a function of height. A region of local strain variation is found near the bottom of the nanowires where growth is initiated in branches at the pore bottoms. Knowledge of how nanoporous templates influences the strain of the nanowires may allow for atomic scale tailoring of the catalytic activity of such nanowires or minimizing strain to optimize electronic device performance.
\end{abstract}


Supplementary material for this article is available online

Keywords: electrodeposition, scanning x-ray diffraction, nanoporous alumina, in situ, strain, strain engineering, catalysis

(Some figures may appear in color only in the online journal)

\section{Introduction}

Nanoscale structures are traditionally defined using lithographic techniques such as UV lithography [1], electron beam lithography [2], and nano-imprint lithography [3]. A costeffective alternative to these traditional lithography methods could be to use nanoporous alumina as templates for ordered arrays of nanodots or nanowires [4, 5]. When aluminum is anodized under certain conditions, the oxide film becomes porous with self-organizing nanopores in a hexagonal pattern [6]. Growth of metal nanowires inside such templates can be performed using electrodeposition, where metal ions from an electrolyte are reduced at the bottom of the pores resulting in the growth of metal nanostructures that adapt the shape of the pores. This has been used to fabricate nanowires of, for example, Ni [7] and $\mathrm{Co}$ [8, 9] for magnetic memories and superconducting Sn nanowires [10]. Pd nanowires have been fabricated and used in applications such as hydrogen sensing [11] and electrocatalysis [12-14].

Nanowires electrodeposited in nanoporous templates grow via a nucleation and growth mechanism. The nucleation rate is governed by the applied cathodic potential, which is the driving force for reducing the Pd ions. The kinetics are governed mostly by mass transport via diffusion and for even slight overpotentials, the reaction is mass-transfer limited [15]. Reports of both 2D plane growth [16] and 3D volume growth [17] of Pd crystallites in nanoporous alumina templates exist in literature. The applied potential can alter the crystallographic growth direction $[9,18]$, exposing different facets to the alumina template walls. This could, in turn, alter the surface energy and the surface stress exerted on the nanowires.

Strain in nanosized-systems influences the catalytic activity both in gas-phase heterogeneous catalysis and electrocatalysis, where tensile and compressive strain have been shown to increase the catalytic activity of different material systems [19-25]. Strain also influence many other physical properties of materials. The bandgap of epitaxially grown semiconductor films can be tuned by growth induced strain due to lattice mismatch [26]. Strain in thin-film semiconductors can also increase the photoluminescence yield due to a modified band structure [27]. In thermoelectric materials, the performance can be increased by strain present in the material [28]. In magnetic metal-organic frameworks, ferroelectric and magnetic order parameters can couple with elastic strain [29]. In $\mathrm{MoS}_{2}$ nanoribbons, the magnetic moment can be increased and tuned by applied strain [30], and the magnetic anisotropy of nanowires can be tuned with strain [31]. It is known that the confinement of the nanoporous template affects the grain size
[32], strain [33], crystallographic structure [34], and crystallographic orientation $[9,32]$. These crystallographic parameters can affect many physical properties of nanostructures, impacting the performance of devices or catalysts. Exactly how the confinement of the nanoporous template affects the growth, strain, and grain size of such metal nanowires is not very well understood. Often metal nanowires grown in nanoporous templates are characterized using transmission electron microscopy (TEM) after the dissolution of the nanoporous template $[13,35-42]$. Altering the surrounding of the nanowires, in this way, may influence the strain state of the nanowires. Due to the lack of non-destructive and in situ studies, there is little knowledge on how growth in the nanoporous templates influences the strain of the nanowires, which in turn influences their physical properties.

We have previously studied the strain in Pd nanowires deposited into nanoporous alumina templates, ex situ, using synchrotron x-ray diffraction [43]. We have also studied strain of Sn nanowires, in situ, during growth using synchrotron scanning x-ray diffraction with a $5 \mu \mathrm{m}$ beam [44]. In this paper, we employ the high flux and penetrative nature of hard $\mathrm{X}$-rays focused to a sub-micron beam $(500 \mathrm{~nm})$ at a modern synchrotron source to follow the growth of Pd nanowires, using scanning $\mathrm{x}$-ray diffraction. The smaller beam size and thinner sample geometry should result in a spatial resolution ten times higher than our previous study. This allows us to study the crystal structure, in situ, during the growth of the nanowires while they are still inside of the nanoporous template. The low cross-section of water in the hard x-ray regime allows for the measurement to be performed in the electrolyte during the growth. Our study reveals a tensile strain that is more pronounced along the growth direction. The strain along the nanowires is also shown to change during the growth process, with local variations in strain in the nanowires close to the position of branches at the bottom of the nanoporous template.

\section{Experimental}

\subsection{Sample preparation}

A hat-shaped sample was machined from a rod of pure polycrystalline aluminum (99.999\%). The sample surface was successively ground using 4000-grit (EU grade) sandpaper, followed by successive polishing down to a $1 \mu \mathrm{m}$ diamond particle suspension (Struers), achieving a mirror-finish. A diamond wire saw was used to cut out a $300 \mu \mathrm{m}$ wide strip 
(a)

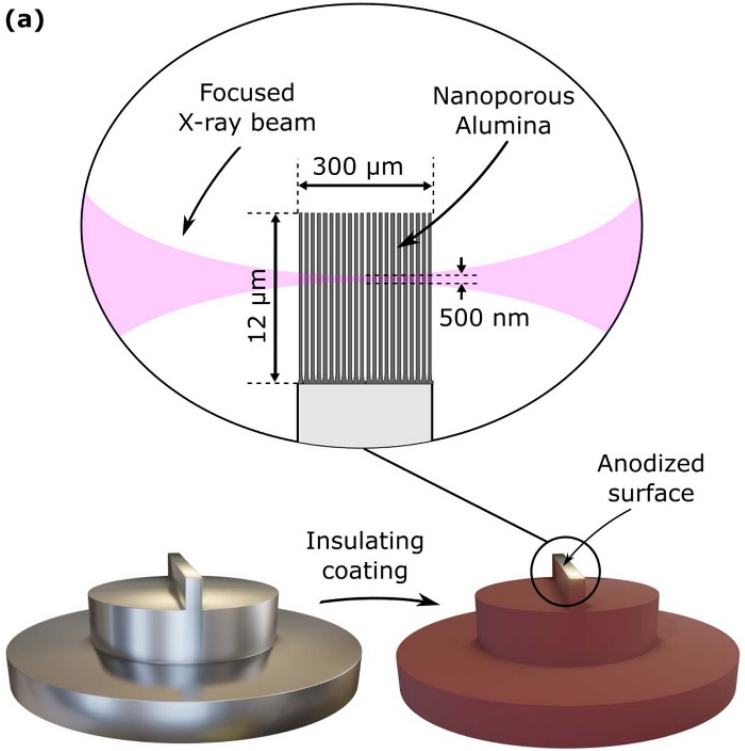

(b)

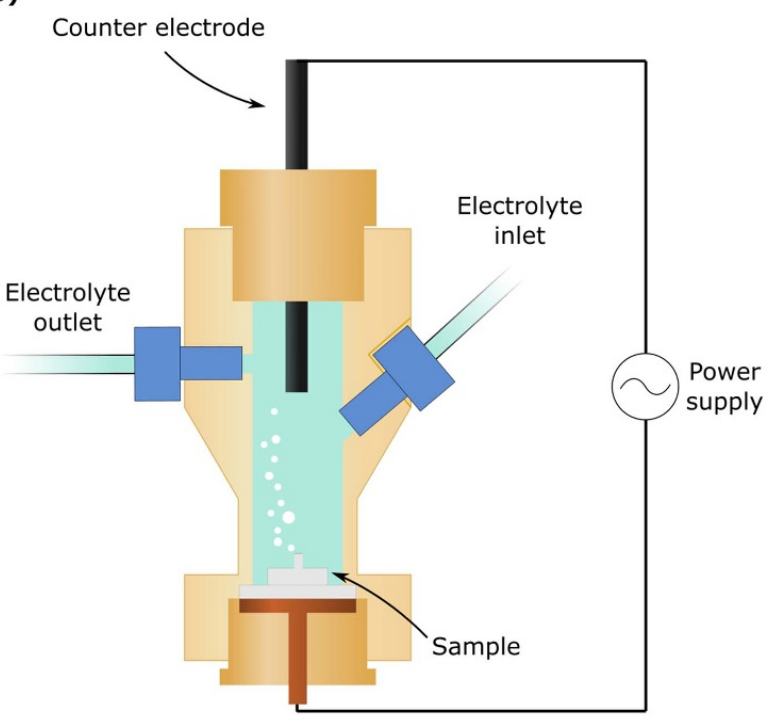

Figure 1. (a) Artistic illustration of sample geometry together with a graphical illustration displaying the focused x-ray beam through the nanoporous film indicating the need for such a sample geometry. (b) Schematic illustration of electrochemical cell dedicated for in situ synchrotron experiments.

on the top of the top-hat-shaped sample. Before anodization, the sample was cleaned by sonication in acetone and ultrapure water. An electrically isolating polymer (nail-varnish) was used to cover the sample and only expose the polished surface on the top of the strip, as shown in figure 1(a). The thin lamella was fabricated to fit within the focus of the x-ray beam, as illustrated in the inset in figure 1(a). The sample was then anodized at $0{ }^{\circ} \mathrm{C}$ in $0.3 \mathrm{M}$ sulfuric acid at $25 \mathrm{~V}$ using a two-step anodization process. The first anodization step was for $10 \mathrm{~h}$, afterwards the sample was submerged in a mixture of chromic acid and phosphoric acid (0.185 $\mathrm{M} \mathrm{CrO}_{3}$ and $0.5 \mathrm{M} \mathrm{H}_{3} \mathrm{PO}_{4}$ ) overnight to dissolve the oxide grown during the first anodization step. The second anodization step was $3 \mathrm{~h}$ long at $25 \mathrm{~V}$ followed by electrochemical barrier layer thinning, where the potential was linearly decreased from $25 \mathrm{~V}$ to $1 \mathrm{~V}$ for $30 \mathrm{~min}$. This process results in a thinner barrier layer, allowing electron tunneling through the oxide layer between the electrolyte and the aluminum substrate. The barrier layer thinning process also results in branching of the pore bottoms.

\subsection{Electrodeposition of Pd nanowires}

Electrodeposition was performed using a neutral electrolyte containing $\mathrm{Pd}\left(\mathrm{NH}_{3}\right)_{4} \mathrm{Cl}_{2}$ and $\mathrm{NH}_{4} \mathrm{Cl}$, which is a well-established electrolyte for $\mathrm{Pd}$ electroplating [45]. A neutral electrolyte is used to avoid dissolution of the porous alumina template during deposition [46]. The exact electrodeposition procedure is described elsewhere [43]. The electrodeposition was performed in a PEEK electrochemical cell dedicated for in situ synchrotron experiments, as shown in figure 1(b). A graphite counter electrode was used, and the electrolyte was continuously pumped to replenish the electroactive $\mathrm{Pd}$ species.

\subsection{In situ scanning $x$-ray diffraction experiment}

The in situ scanning X-ray diffraction experiment was performed at the 'In situ and Nano Diffraction' beamline, P23, at PETRA III, DESY in Hamburg, Germany. A sub-micron focused beam with a vertical spot size of $\sim 500 \mathrm{~nm}$ at the sample position was used. The $\mathrm{x}$-ray energy was $18.275 \mathrm{keV}$. The electrochemical cell was mounted on a $(5+2)$ circle HUBER diffractometer equipped with a Eulerian cradle, as shown in the supplementary information (figure S1 (available online at stacks.iop.org/JPD/54/235301/mmedia)). An $\mathrm{X}$-Spectrum Lambda $750 \mathrm{~K}$ detector with a pixel size of $55 \mu \mathrm{m}$ and active area of $512 \times 1528$ pixels was used at a sampledetector distance of $360 \mathrm{~mm}$, resulting in an angular resolution of $0.01^{\circ}$. The diffractometer was calibrated using an in-house $\mathrm{LaB}_{6}$ standard. The sub-micron focused x-ray beam impinged on the sample in a transmission geometry, where the beam was parallel to the sample surface. To gain structural information along the nanowires, during the growth, the sample and the electrochemical cell were scanned through the beam along the growth direction of the nanowires, as shown in the schematics of figures 2(a) and (b). The intensity of the direct beam together with the palladium diffraction signal as a function of height, while the sample was scanned through the beam, can be found in the supplementary information (figure $\mathrm{S} 2(\mathrm{a})$ ). The diffraction signal was recorded in and out of the sample surface plane. Detector images measured in-and outof-plane, before and at the end of the deposition, are shown in figure 2(c), illustrating the diffraction powder rings that were measured on the area detector.

\subsection{Data analysis}

1D diffraction patterns were generated from the 2D detector images by assigning each pixel a $\delta$ and $\gamma$ value as described 


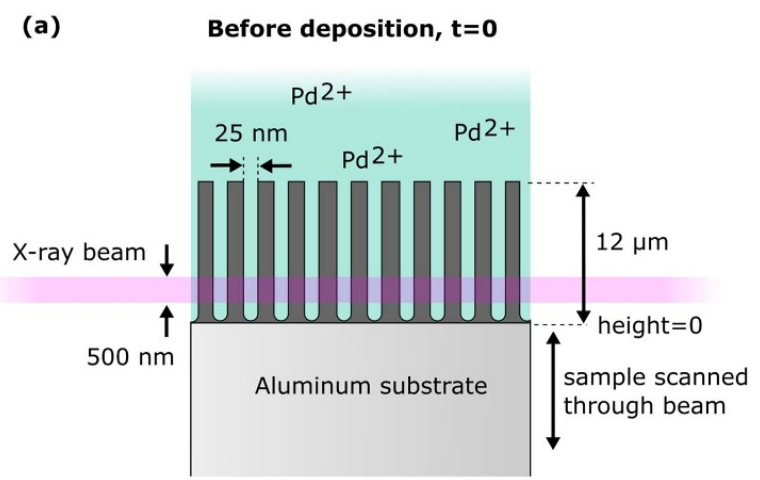

(c)

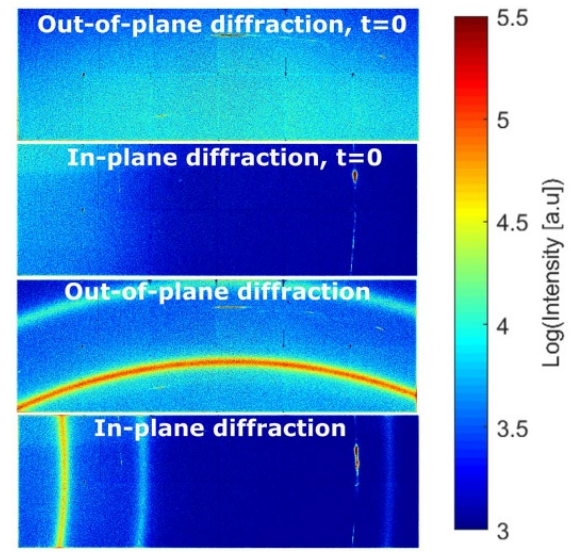

(b) End of deposition, $\mathbf{t}=55 \mathrm{~min}$

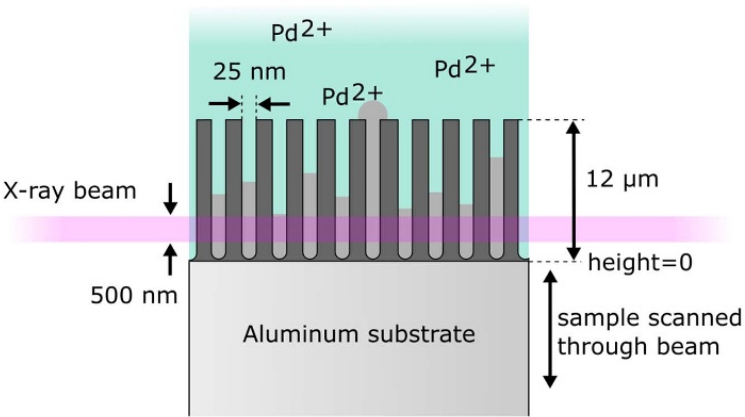

(d)
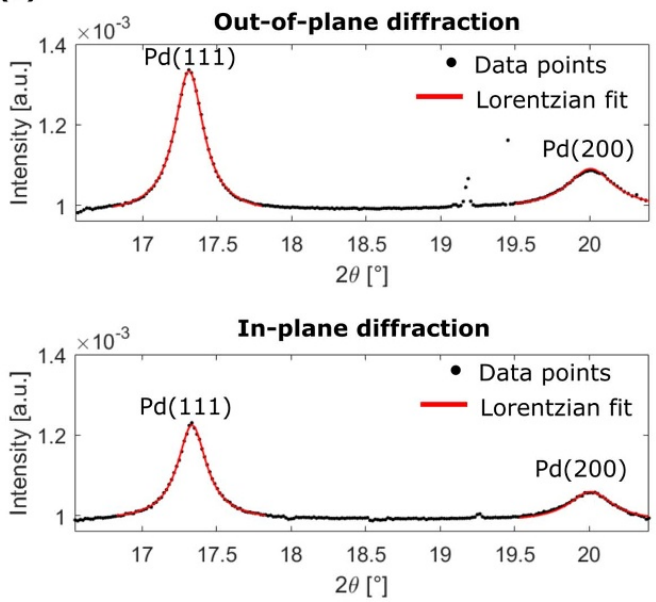

Figure 2. (a) Illustration of the sample before the deposition of Pd. (b) Illustration of the sample at the end of the deposition. (c) Detector images at sample height $=1 \mu \mathrm{m}$ before and at the end of the deposition of Pd nanowires. (d) Lorentzian fitting of diffraction data in-and out-of-plane.

by Schlepütz et al [47], then $2 \theta$ for each pixel was calculated as $2 \theta=\arccos \left(\cos \left[\gamma_{\text {pixel }}\right] \times \cos \left[\delta_{\text {pixel }}\right]\right)$. The $\operatorname{Pd}(111)$ and (200) peaks were fitted with a Lorentzian line profile, as shown in figure 2(d). The size-contribution to the peak line broadening is Lorentzian [48]. Since the instrumental resolution and bandwidth of modern synchrotrons are negligible compared to the size broadening from nanometer-sized crystallites, which is the case for nanowires grown in nanoporous alumina, a pure Lorentzian profile was used to fit the peaks. The peak position and FWHM were used to calculate the lattice strain and average grain size along and across the nanowires. The horizontal size of the $\mathrm{x}$-ray beam was much larger than the diameter of the nanowires of $25 \mathrm{~nm}$. Therefore no spatial information could be obtained perpendicular to the nanowire growth direction. The beam intersection across the lamella contains approximately 4000 nanopores. The resulting diffraction pattern is hence an average of many nanowires.

Lattice strain for a specific set of atomic planes was calculated as shown in the equation:

$$
\varepsilon_{\mathrm{hkl}}=\left(d_{\mathrm{hkl}}-d_{\mathrm{hkl}}^{0}\right) / d_{\mathrm{hkl}}^{0}
$$

where $d_{\mathrm{hkl}}$ is the lattice plane spacing for a certain set of planes with miller indices hkl and $d_{\mathrm{hkl}}^{0}$ is here taken as the equivalent lattice plane spacing in bulk Pd with the lattice parameter of
$3.98 \AA$. The grain size was calculated from the line broadening using Scherrer's formula. Diffraction in the sample surface plane, where the $Q$ vector is perpendicular to the growth direction, gives information about lattice strain and grain size perpendicular to the growth direction. Diffraction measured out-of-plane gives information about lattice strain and grain size along the $Q$ vector, which is oriented with an angular offset from the surface normal equal to the Bragg angle $\theta$. We do not measure strain and grain size exactly along the growth direction of the nanowires but with an offset of $8.5^{\circ}$ for the $\operatorname{Pd}(111)$ out-of-plane reflection and an offset of $10^{\circ}$ for the $\operatorname{Pd}(200)$ out-of-plane reflection.

\section{Results}

To study the strain state of the nanowires in situ and with spatial resolution, scanning $\mathrm{x}$-ray diffraction was used, which resulted in a $3 \mathrm{D}$ data set of spatial, reciprocal and time coordinates. From the scanning diffraction dataset, we can visualize the growth of the nanowires over time by considering the $\operatorname{Pd}(111)$ Bragg reflection as a function of height at different time stamps, as shown in figure 3. Figure 3(a) shows the outof-plane $\operatorname{Pd}(111)$ Bragg reflection as a function of height (vertical direction) for several time steps, and (b) similarly shows 
(a)

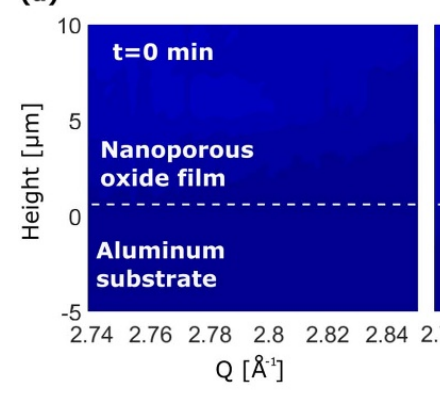

(b)
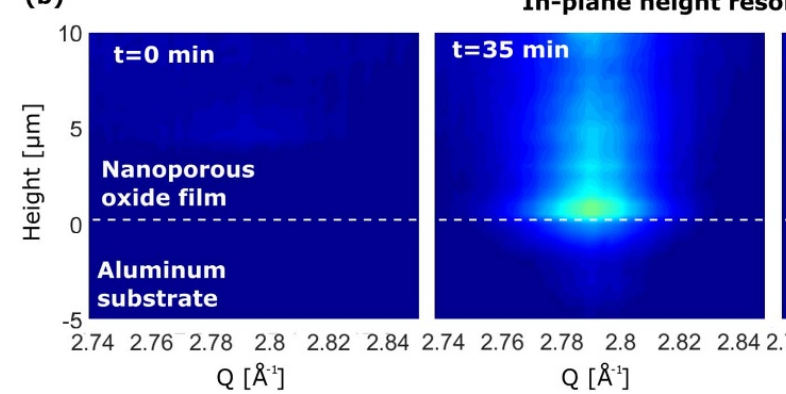

$\mathrm{Q}\left[\AA^{-1}\right]$

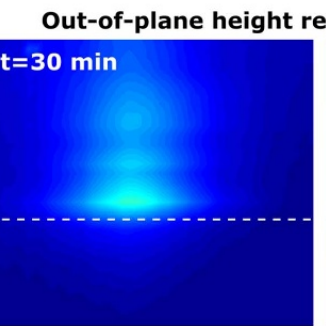

$\mathrm{Q}\left[\AA^{-1}\right]$

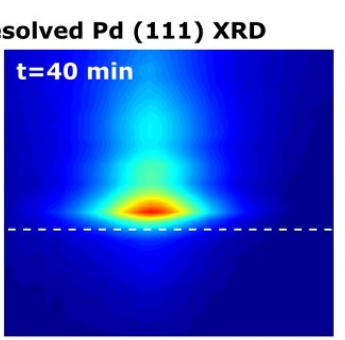

$\mathrm{Q}\left[\AA^{-1}\right]$

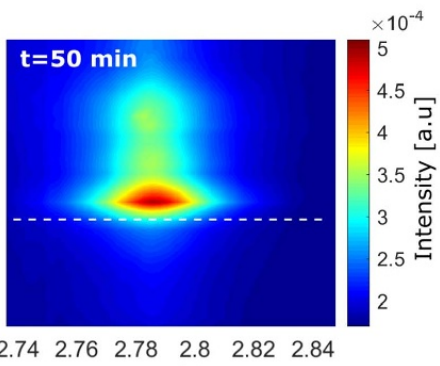

$\mathrm{Q}\left[\AA^{-1}\right]$

Figure 3. (a) The evolution of the $\mathrm{Pd}(111)$ out-of-plane diffraction as a function of sample height at several times during the electrodeposition of $\mathrm{Pd}$. (b) $\mathrm{Pd}(111)$ in-plane diffraction as a function of height at several times during electrodeposition of Pd.

the in-plane Pd(111) Bragg reflection. The in-and out-of-plane diffraction, at the sample height of $1 \mu \mathrm{m}$, is shown as a function of time in the supplementary information (figure S2(b)). During the first 25 min of the growth, the signal was too weak to analyze. Therefore that data were excluded from the analysis. As seen, the growth of the nanowires is initiated at the bottom of the nanopores at height 0 . Over time, the $\mathrm{Pd}(111)$ intensity profile grows taller indicating growth of crystalline Pd up through the pores. At all times, the intensity of the diffraction signal is the strongest at the bottom of the pores and there is a gradient in diffraction intensity as a function of height. This could be explained by an uneven height distribution of the nanowires during the growth, with some nanowires being taller than others.

Microscopy techniques, such as SEM, have been widely used to study the morphology of nanowires deposited into nanoporous alumina templates [39-42]. Even if destructive preparation is required, such as focused ion beam milling, it is a very useful method for visualization of the nanowires inside of the template. With electron microscopy, we have observed branches at the bottom of Pd nanowires grown in nanoporous templates in a previous study [43]. The barrier layer thinning step in the fabrication of the nanoporous alumina template results in branching of the pore bottoms. This results in branching at the bottom of the nanowires grown in the templates, which is well documented in the literature $[35,49,50]$. An SEM image of the branched pore bottoms can be found in the supplementary information (figure S3). It has been reported for $\mathrm{Sn}$ nanowires that growth through the branched pore bottoms affects the growth mode of the nanowires and results in a variation in grain size, strain and mosaicity [44].

To calculate the lattice strain of the nanowires as a function of height, the peak position was extracted by fitting the
Brag peaks with a Lorentzian line profile as seen in figure 2(d). The lattice strain in-and out-of-plane (along and across the nanowires) was calculated from the position of the $\operatorname{Pd}(111)$ reflection since it is the most intense reflection. The lattice parameter of bulk Pd, $a=3.89 \AA$, was used as a reference to calculate the lattice strain, $\varepsilon$, as shown in equation (1). An illustration of the orientation of the lattice planes probed in the nanowires during out-of-plane and in-plane diffraction is shown in figure 4(a). During out-of-plane diffraction, the $Q$ vector is nearly aligned with the growth direction and hence lattice strain is measured along the growth direction. During in-plane diffraction, the $Q$ vector is oriented perpendicular to the growth direction. Therefore the strain measured in-plane is oriented perpendicular to the growth direction, which is the confined direction of the template. Figure 4(b) shows the local average strain of the Pd nanowires along the out-of-plane direction (along the nanowires) as a function of height and time. Figure 4(c) shows the local average strain of the Pd nanowires along the in-plane direction (across the nanowires) as a function of height and time.

The strain measured in-and out-of-plane shows clear variations along the wires and it also varies at the different stages of the growth. The strain measured out-of-plane, as shown in figure 4(b), is tensile in the nanowires compared to that of bulk Pd. Just above the bottom of the pores, marked by a dotted black line in the figure, a region of less strain can be seen, which is present at all three stages of the growth measured. Above this region, there is a tensile strain in the nanowires that is increasing as the nanowires grow. Also, in the in-plane direction there is a variation in the strain along the nanowires, as seen in figure 4(c). The lattice strain across the nanowires is also tensile but to a lesser degree than the strain measured out-of-plane. Above the bottom of the pores, there is a region 
(a)

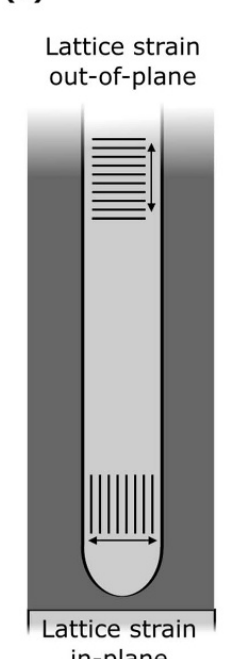

(d)

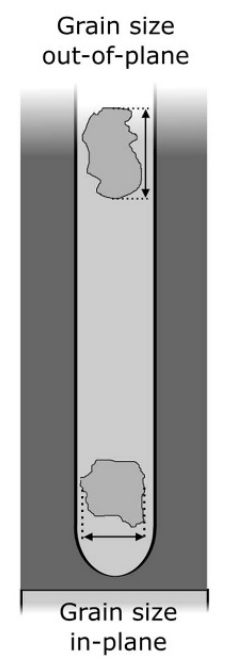

(b)

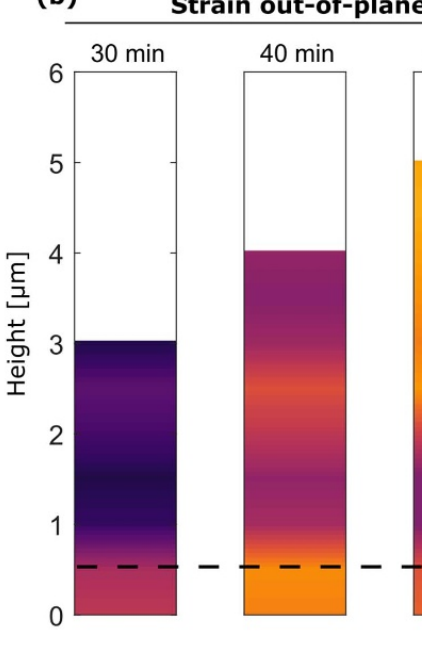

(e)

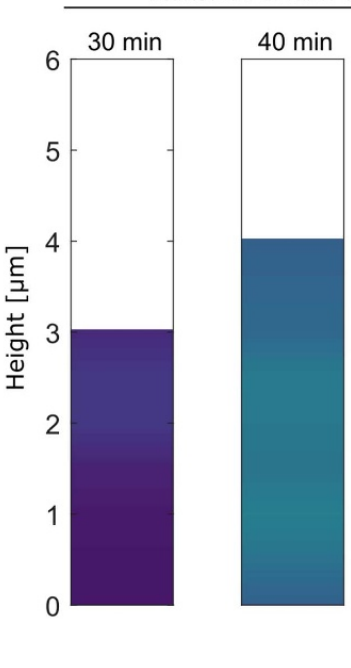

(c) Strain in-plane

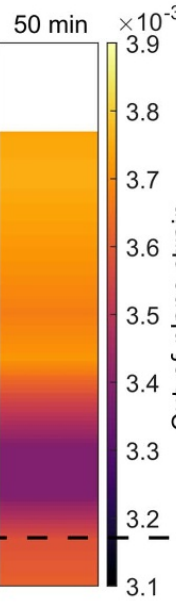

3.8

莒

$\frac{\stackrel{0}{\frac{\pi}{2}}}{\frac{\pi}{2}}$

ㄴ.

今

Above

pore

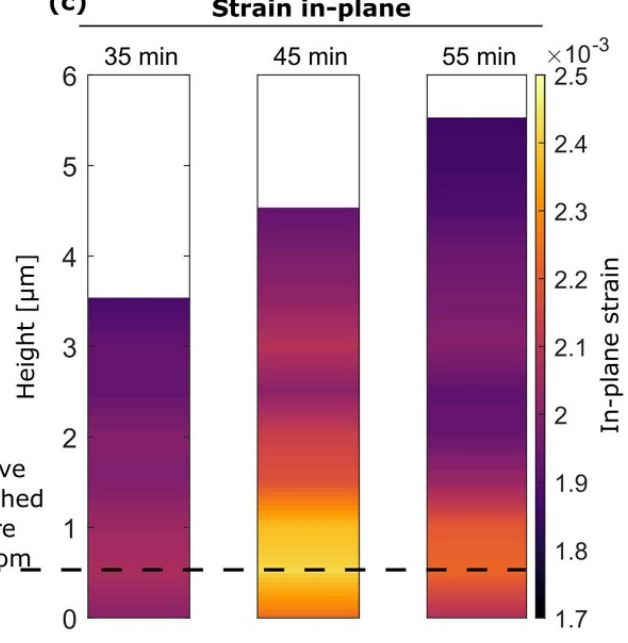

(f)

Grain size in-plane

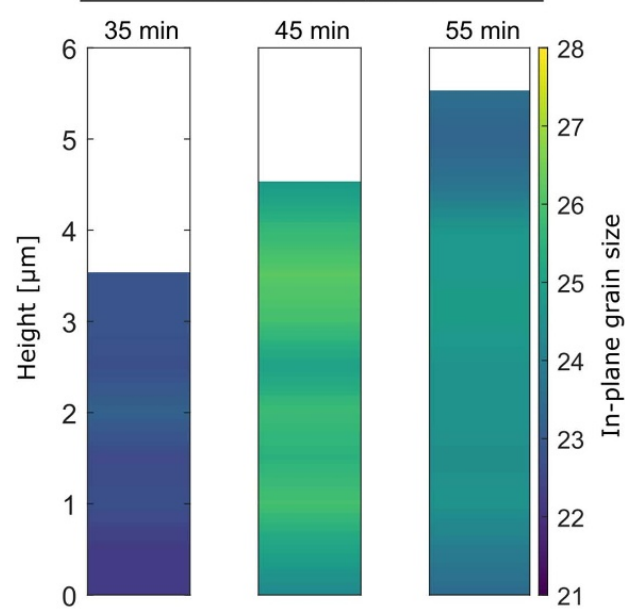

Figure 4. Spatially resolved structural information obtained from in situ XRD measurements at three different deposition times.

(a) Illustration of the lattice strain directions measured in the nanowires. (b) Variation in out-of-plane lattice strain along the nanowires.

(c) Variation in in-plane strain along the nanowires. (d) Illustration of grain size for different directions measured in the nanowires.

(e) Grain size along the nanowires measured out-of-plane. (f) Grain size along the nanowires measured in-plane.

of more tensile strain. The strain along the growth direction is more tensile than across the nanowires, which is the confined direction. The lattice is hence more expanded in the direction which is not confined by the nanoporous template. This illustrates an effect on the crystal structure imposed by the nanoconfinement of the templates. A point to notice here is that the trends for the strain in-and out-of-plane are each other's opposite. When there is higher strain in-plane, there is less strain out-of-plane and vice versa. When comparing the strain at $0.5 \mu \mathrm{m}$, just above the branched pore bottoms, marked by a dotted black line in the figure, there is a variation in the strain as time proceeds during the growth process. This indicates that lattice strain can develop and decrease in regions of the nanowires that are already grown. Little growth occurs at the height of $0.5 \mu \mathrm{m}$ during the times shown, as most pores are already filled to this point. Inside the pores, the growth takes place at the top of the nanowires, where Pd ions are electrochemically reduced from the electrolyte and incorporated in the lattice. Yet, there is still strain developing close to the bottom of the pores during the growth process.

The grain size of the nanowires was calculated from the line broadening using Scherrer's formula. Figure 4(d) shows a schematic illustration of the grain sizes in the nanowires probed during in-and out-of-plane diffraction, calculated from the line broadening. Figure 4(e) shows the local average size of the crystalline grains along the out-of-plane direction (along the nanowires) as a function of height at various times. Similarly, figure 4(f) shows the local average size of the crystalline grains along the in-plane direction (across the nanowires), also as a function of height. The average size of the grains both inand out-of-plane is around $25 \mathrm{~nm}$, matching the diameter of the nanoporous template of $25 \mathrm{~nm}$. As time proceeds during the deposition and more growth occur, an increase in the grain size is observed along the wires. It can also be seen that the grains are slightly smaller at the bottom of the pores, where the nanowires grow up through the branched pore bottoms. 
The branched pore bottoms have a smaller pore diameter, so the crystallite size is restricted and is expected to be smaller, as observed.

\section{Discussion}

The results presented in this paper were made possible by the recent technical advances at synchrotron facilities with brighter sources and access to intense nano-focused beams in the tender to hard x-ray regime, which opens for new kinds of experiments [51, 52]. A large benefit of X-rays compared to electron-based techniques, such as TEM and SEM, is the penetrative nature of X-rays, which allows for studies to be performed on thicker embedded structures as well as in situ and in operando conditions. With X-rays, it is possible to probe and measure inside batteries [53], embedded transistors [54], and during semiconductor nanoscale growth [55]. With new nano-focused x-ray beamlines, it will be possible to push the spatial resolution down to tens of nanometers and extract chemical information using fluorescence [56] or x-ray absorption mapping. Spatially resolved structural information, as demonstrated here, is accessible with scanning x-ray diffraction. A further strength of these recent advances in the brightness of synchrotron sources is the possibility to reduce the exposure time, allowing us to push the temporal resolution of the experiments. In summary, the penetrative nature of nano-focused beams allows experiments to be performed in situ with increased spatial and temporal resolution, where both chemical and structural information can be extracted.

We have shown that we can follow the process of electrochemical crystal growth in nanoconfined templates, in situ. With a sub-micron beam, we obtain spatial information about the grain size and lattice strain across and along the growth direction of the nanowires. We have shown that the crystal growth is initiated at the bottom of the pores and the nanowires grow up through the template as a function of deposition time. The grain size measured is around $25 \mathrm{~nm}$, which is the same as the pore diameter, thus indicating that the crystallite size is limited by the nanoconfinement inside the template. With the spatial resolution achieved via scanning diffraction, we could observe that the grain size is smaller near the bottom of the pores, indicating that the grains are smaller when growing through the branches. The scanning diffraction technique allows for strain variations along the nanowires to be visualized at different deposition times. Above the bottom branched part of the pores, there is an evident variation in the strain showing less tensile strain out-of-plane and more tensile strain in-plane. This indicates an effect of the nanoconfined template on the strain state of the nanowires. The strain here also varies as the nanowires grow taller over time. Therefore, it is shown that stress can propagate through the crystalline nanowire as growth proceeds. Measurements performed ex situ would not be able to capture this phenomenon of strain development during the growth process. Post characterization using other techniques, such as TEM, is very common after the dissolution of the template. But this might reveal a different story about the strain state of the nanowires if the stress caused by the nanoconfinement is released upon dissolution of the template.

Our finding of a tensile lattice strain of the Pd crystallites in the nanowires is similar to reports from Shin et al, who reported, experimentally, a close-to linear relationship between the lattice parameter along the growth direction and the inverse of $\mathrm{Sn}$ nanowire radius [33]. This was shown to be true not only for single-crystalline but also for polycrystalline wires [57]. A nano-sized template gives rise to a larger lattice parameter in the growth direction compared to the bulk material, hence more tensile strain. In later studies, Shin et al attribute this to a compressive growth stress acting perpendicular to the growth direction in the confined direction of the template [58]. This causes tensile strain in the growth direction and a relative compressive strain in the confined direction due to the Poisson effect. This prediction is in line with our previous study of $\mathrm{Pd}$ nanowires, where we measure the strain both along and perpendicular to the growth direction [43] while Shin's claim of a compressive growth stress is based on a theoretical approach. In our present study, we do not only support Shin's model, but we also show that there are variations in the tensile strain along the nanowires.

Both our previous ex situ Pd nanowire study [43] and our in situ study of Sn deposition [44] showed a difference in the strain between the growth direction and in-plane direction of $\sim 0.5 \%$. However, the magnitude of strain was different where we found a strain of $\sim 1.2 \%$ for the Sn nanowires and $\sim 0.3 \%$ in the case of Pd. This indicates that different effects play a role in the strain state of the nanowires that are both material-dependent and independent. Pd has a surface free energy almost three times as large as Sn [59], leading to a greater driving force to minimize the surface of the $\mathrm{Pd}$ nanowires compared to the case of $\mathrm{Sn}$. However, the relative in-plane compression was comparable between the two cases suggesting that the compressive stress exerted by the nanoconfined template is less material dependent.

A new observation, not present in our previous ex situ study, is that the lattice parameters are larger when measured in situ. This difference in the observed lattice parameters could be due to lattice expansion from hydrogen absorption during the electrodeposition. Such an effect would not be detected in our ex situ measurements due to the desorption of hydrogen in the absence of a high external hydrogen partialpressure. It is known that during electrodeposition, the potential is high enough to electrochemically evolve hydrogen at the metal electrolyte interface [60]. Therefore an increased hydrogen partial pressure is expected. Pd has a large capacity to store hydrogen [61], which could explain the larger tensile strain observed during this in situ study. Hydrogen absorption could also explain the observed strain evolution in the nanowires during the deposition.

\section{Conclusions}

Understanding how growth in nanoconfined templates influences strain, grain size, and other crystallographic parameters is of importance for templated nanostructures to be 
successfully used in future devices. We have demonstrated that we could follow the growth process of the nanowires and extract information about the grain size and strain along the nanowires during the growth process. We found that there is a tensile strain in the nanowires and that it is more pronounced along the growth direction than in the confined direction of the templates. It was also found that the lattice is more expanded during the in situ measurement compared to our previous ex situ study. This is attributed to the absorption of electrochemically evolved hydrogen in the Pd lattice. With the spatial information made possible with the focused $\mathrm{x}$-ray beam we could observe local variations in strain as a function of height. A region of local strain variation is found near the bottom of the nanowires where growth is initiated in branches at the pore bottoms. This indicates that the branched pore bottoms give rise to local strain in the nanowires. The knowledge of how nanoporous templates influences the strain of the grown nanowires may allow for the atomic scale tailoring of such nanowires. This knowledge of strain induced by growth in nanoporous templates can also be expanded beyond the scope of noble metal catalysts. Strain engineering is also used for magnetic materials [62], solar cells [63], and energy storage [64].

\section{Acknowledgments}

We acknowledge DESY (Hamburg, Germany), a member of the Helmholtz Association HGF, for the provision of experimental facilities. Parts of this research were carried out at PETRA III and we would like to thank Azat Khadiev for assistance in using beamline P23. This work was financially supported by the Swedish Research Council through the Röntgen-Ångström Cluster 'In-situ High Energy X-ray Diffraction from Electrochemical Interfaces (HEXCHEM)' (Project No. 2015-06092), by the 'Atomic Resolution Cluster'a Research Infrastructure Fellow program of the Swedish Foundation for Strategic Research, and project grant 'Understanding and Functionalization of Nano Porous Anodic Oxides' (Project No. 2018-03434) by the Swedish research council. We acknowledge financial support by NanoLund.

\section{ORCID iDs}

Alfred Larsson (D) https://orcid.org/0000-0002-8932-8381 Giuseppe Abbondanza (D) https://orcid.org/0000-0002-06805454

Edvin Lundgren (D) https://orcid.org/0000-0002-3692-6142

Gary S Harlow (D) https://orcid.org/0000-0001-6644-0648

\section{References}

[1] Fu N, Liu Y, Ma X and Chen Z 2019 EUV lithography: state-of-the-art review J. Microelectron. Manuf. 2 1-6

[2] Chen Y 2015 Nanofabrication by electron beam lithography and its applications: a review Microelectron. Eng. 135 57-72

[3] Schift H 2008 Nanoimprint lithography: an old story in modern times? A review J. Vac. Sci. Technol. B 26 458-80
[4] Sousa C T, Leitao D C, Proenca M P, Ventura J, Pereira A M and Araujo J P 2014 Nanoporous alumina as templates for multifunctional applications Appl. Phys. Rev. 17-8

[5] Lee W and Park S J 2014 Porous anodic aluminum oxide: anodization and templated synthesis of functional nanostructures Chem. Rev. 114 7487-556

[6] Sulka G 2008 Highly Ordered Anodic Porous Alumina Formation by Self-Organized Anodizing (Germany: Wiley-VCH Verlag GmbH \& Co. KGaA) pp 1-116

[7] Nielsch K, Wehrspohn R B, Barthel J, Kirschner J, Gosele U, Fischer S F and Kronmüller H 2001 Hexagonally ordered $100 \mathrm{~nm}$ period nickel nanowire arrays Appl. Phys. Lett. 79 1360-2

[8] Zhang J, Jones G A, Shen T H, Donnelly S E and Li G H 2007 Monocrystalline hexagonal-close-packed and polycrystalline face-centered-cubic Co nanowire arrays fabricated by pulse dc electrodeposition J. Appl. Phys. 101

[9] Wang M, Wu Z, Yang H and Liu Y 2018 Growth orientation control of Co nanowires fabricated by electrochemical deposition using porous alumina templates Cryst. Growth Des. 18 479-87

[10] Tian M L, Wang J G, Snyder J, Kurtz J, Liu Y, Schiffer P, Mallouk T E and Chan M H W 2003 Synthesis and characterization of superconducting single-crystal $\mathrm{Sn}$ nanowires Appl. Phys. Lett. 83 1620-2

[11] Kim K T, Sim S J and Cho S M 2006 Hydrogen gas sensor using Pd nanowires electro-deposited into anodized alumina template IEEE Sens. J. 6 509-13

[12] Xu C W, Wang H, Shen P K and Jiang S P 2007 Highly ordered Pd nanowire arrays as effective electrocatalysts for ethanol oxidation in direct alcohol fuel cells $A d v$. Mater. 194256

[13] Cheng F L, Wang H, Sun Z H, Ning M X, Cai Z Q and Zhang M 2008 Electrodeposited fabrication of highly ordered Pd nanowire arrays for alcohol electrooxidation Electrochem. Commun. 10 798-801

[14] Slaughter G and Kulkarni T 2016 Fabrication of palladium nanowire array electrode for biofuel cell application Microelectron. Eng. 149 92-96

[15] Bograchev D A, Volgin V M and Davydov A D 2013 Simple model of mass transfer in template synthesis of metal ordered nanowire arrays Electrochim. Acta 96 1-7

[16] Zhang H, Jia W, Sun H, Guo L and Sun J 2018 Growth mechanism and magnetic properties of Co nanowire arrays by AC electrodeposition J. Magn. Magn. Mater. 468 188-92

[17] Tasaltin N, Ozturk S, Kilinc N, Yuzer H and Ozturk Z Z 2010 Fabrication of vertically aligned Pd nanowire array in AAO template by electrodeposition using neutral electrolyte Nanoscale Res. Lett. 5 1137-43

[18] Stegmann C, Muench F, Rauber M, Hottes M, Brötz J, Kunz U, Lauterbach S, Kleebe H-J and Ensinger W 2014 Platinum nanowires with pronounced texture, controlled crystallite size and excellent growth homogeneity fabricated by optimized pulsed electrodeposition RSC Adv. 4 4804-10

[19] Jansonius R P, Schauer P A, Dvorak D J, MacLeod B P Fork D K and Berlinguette C P 2020 Strain influences the hydrogen evolution activity and absorption capacity of palladium Angew. Chem., Int. Ed. Engl. 59 12192-8

[20] Sun W, Wang Z, Zaman W Q, Zhou Z, Cao L, Gong X Q and Yang J 2018 Effect of lattice strain on the electro-catalytic activity of $\mathrm{IrO}_{2}$ for water splitting Chem. Commun. 54 996-9

[21] Wang L, Zeng Z, Gao W, Maxson T, Raciti D, Giroux M, Pan X, Wang C and Greeley J 2019 Tunable intrinsic strain in two-dimensional transition metal electrocatalysts Science $363870-4$

[22] Westsson E, Picken S and Koper G 2019 The effect of lattice strain on catalytic activity Chem. Commun. 55 1338-41 
[23] Xia Z and Guo S 2019 Strain engineering of metal-based nanomaterials for energy electrocatalysis Chem. Soc. Rev. 48 3265-78

[24] Yan K, Maark T A, Khorshidi A, Sethuraman V A, Peterson A A and Guduru P R 2016 The influence of elastic strain on catalytic activity in the hydrogen evolution reaction Angew. Chem., Int. Ed. Engl. 55 6175-81

[25] Sebastián-Pascual P, Mezzavilla S, Stephens I E L and Escudero-Escribano M 2019 Structure-sensitivity and electrolyte effects in $\mathrm{CO}_{2}$ electroreduction: from model studies to applications ChemCatChem 11 3626-45

[26] Dong L, Yadav S K, Ramprasad R and Alpay S P 2010 Band gap tuning in $\mathrm{GaN}$ through equibiaxial in-plane strains Appl. Phys. Lett. 96202106

[27] Ahn G H, Amani M, Rasool H, Lien D-H, Mastandrea J P, Ager Iii J W, Dubey M, Chrzan D C, Minor A M and Javey A 2017 Strain-engineered growth of two-dimensional materials Nat. Commun. 8608

[28] Wu Y, Chen Z, Nan P, Xiong F, Lin S, Zhang X, Chen Y, Chen L, Ge B and Pei Y 2019 Lattice strain advances thermoelectrics Joule 3 1276-88

[29] Xin L, Zhang Z, Carpenter M A, Zhang M, Jin F, Zhang Q, Wang X, Tang W and Lou X 2018 Strain coupling and dynamic relaxation in a molecular perovskite-like multiferroic metal-organic framework Adv. Funct. Mater. 281806013

[30] Pan H and Zhang Y-W 2012 Tuning the electronic and magnetic properties of $\mathrm{MoS}_{2}$ nanoribbons by strain engineering J. Phys. Chem. C 116 11752-7

[31] Lei $\mathrm{N}$ et al 2013 Strain-controlled magnetic domain wall propagation in hybrid piezoelectric/ferromagnetic structures Nat. Commun. 41378

[32] Dalchiele E A, Marotti R E, Cortes A, Riveros G, Gomez H, Martinez L, Romero R, Leinen D, Martin F and Ramos-Barrado J R 2007 Silver nanowires electrodeposited into nanoporous templates: study of the influence of sizes on crystallinity and structural properties Physica E 37 184-8

[33] Shin H S, Yu J, Song J Y and Park H M 2009 Size dependence of lattice deformation induced by growth stress in $\mathrm{Sn}$ nanowires Appl. Phys. Lett. 94

[34] Liu X H, Luo J and Zhu J 2006 Size effect on the crystal structure of silver nanowires Nano Lett. 6 408-12

[35] Xu L P, Yuan Z H and Zhang X G 2006 Fabrication of multi-level branched metal nanowires by AAO template electrodeposition Chin. Sci. Bull. 51 2055-8

[36] Li L, Yang Y W, Huang X H, Li G H and Zhang L D 2006 Pulsed electrodeposition of single-crystalline $\mathrm{Bi}_{2} \mathrm{Te}_{3}$ nanowire arrays Nanotechnology 17 1706-12

[37] Zhang X R, Hao Y F, Meng G W and Zhang L D 2005 Fabrication of highly ordered InSb nanowire arrays by electrodeposition in porous anodic alumina membranes J. Electrochem. Soc. 152 C664-8

[38] Zsurzsa S, Pellicer E, Sort J, Peter L and Bakonyi I 2018 Electron microscopy characterization of electrodeposited homogeneous and multilayered nanowires in the $\mathrm{Ni}-\mathrm{Co}-\mathrm{Cu}$ system J. Electrochem. Soc. 165 D536-42

[39] Dost R, Zhou Y, Zhang H, Allwood D A and Inkson B J 2020 Effect of annealing on the electrical and magnetic properties of electrodeposited $\mathrm{Ni}$ and permalloy nanowires J. Magn. Magn. Mater. 499

[40] Sauer G, Brehm G, Schneider S, Nielsch K, Wehrspohn R B, Choi J, Hofmeister H and Gösele U 2002 Highly ordered monocrystalline silver nanowire arrays J. Appl. Phys. $913243-7$

[41] Napolskii K S, Roslyakov I V, Eliseev A A, Petukhov D I, Lukashin A V, Chen S F, Liu C-P and Tsirlina G A 2011 Tuning the microstructure and functional properties of metal nanowire arrays via deposition potential Electrochim. Acta 56 2378-84

[42] Barriga-Castro E D, Garcia J, Mendoza-Resendez R, Prida V M and Luna C 2017 Pseudo-monocrystalline properties of cylindrical nanowires confinedly grown by electrodeposition in nanoporous alumina templates $R S C$ Adv. 7 13817-26

[43] Larsson A, Abbondanza G, Linpé W, Carlà F, Mousley P, Hetherington C, Lundgren E and Harlow G S 2020 Electrochemical fabrication and characterization of palladium nanowires in nanoporous alumina templates J. Electrochem. Soc. 167122514

[44] Harlow G S, Drnec J, Wiegmann T, Lipe W, Evertsson J, Persson A R, Wallenberg R, Lundgren E and Vinogradov N A 2019 Observing growth under confinement: $\mathrm{Sn}$ nanopillars in porous alumina templates Nanoscale Adv. 1 4764-71

[45] Abys J 2011 Modern Electroplating 5th edn (Germany: Wiley-VCH Verlag GmbH \& Co. KGaA) pp 327-68

[46] Mardilovich P P, Govyadinoy A N, Mazurenko N I and Paterson R 1995 New and modified anodic alumina membranes part II. Comparison of solubility of amorphous (normal) and polycrystalline anodic alumina membranes J. Memb. Sci. 98 143-55

[47] Schleputz C M, Mariager S O, Pauli S A, Feidenhans'l R and Willmott P R 2011 Angle calculations for a (2+3)-type diffractometer: focus on area detectors $J$. Appl. Crystallogr. $4473-83$

[48] Cox D E 1991 Powder diffraction Handbook on Synchrotron Radiation vol 3 (Netherlands: North Holland) pp 157-96

[49] Stepniowski W J, Florkiewicz W, Michalska-Domanska M, Norek M and Czujko T 2015 A comparative study of electrochemical barrier layer thinning for anodic aluminum oxide grown on technical purity aluminum J. Electroanal. Chem. 741 80-86

[50] Wu Z, Zhang Y W and Du K 2013 A simple and efficient combined AC-DC electrodeposition method for fabrication of highly ordered Au nanowires in AAO template Appl. Surf. Sci. 265 149-56

[51] Johansson U, Vogt U and Mikkelsen A 2013 NanoMAX: a hard x-ray nanoprobe beamline at MAX IV Proc. SPIE Optical Engineering + Applications $\mathbf{8 8 5 1}$

[52] Wright J, Giacobbe C and Majkut M 2020 New opportunities at the materials science beamline at ESRF to exploit high energy nano-focus x-ray beams Curr. Opin. Solid State Mater. Sci. 24100818

[53] Bak S-M, Shadike Z, Lin R, Yu X and Yang X-Q 2018 In situ/operando synchrotron-based $\mathrm{x}$-ray techniques for lithium-ion battery research NPG Asia Mater. 10 563-80

[54] Dzhigaev D et al 2020 Strain mapping inside an individual processed vertical nanowire transistor using scanning x-ray nanodiffraction Nanoscale 12 14487-93

[55] Takahasi M 2018 In situ synchrotron x-ray diffraction study on epitaxial-growth dynamics of III-V semiconductors Japan. J. Appl. Phys. 57050101

[56] Linpe W, Harlow G S, Evertsson J, Hejral U, Abbondanza G, Lenrick F, Seifert S, Felici R, Vinogradov N A and Lundgren E 2019 The state of electrodeposited Sn nanopillars within porous anodic alumina from in situ x-ray observations ACS Appl. Nano Mater. 2 3031-8

[57] Shin H S, Yu J, Song J Y and Park H M 2011 Size-dependent lattice parameters of microstructure-controlled $\mathrm{Sn}$ nanowires J. Mater. Res. 26 2033-9

[58] Shin H S, Yu J, Song J Y, Park H M and Kim Y-S 2010 Origins of size-dependent lattice dilatation in tetragonal $\mathrm{Sn}$ nanowires: surface stress and growth stress Appl. Phys. Lett. 97131903 
[59] Vitos L, Ruban A V, Skriver H L and Kollár J 1998 The surface energy of metals Surf. Sci. 411 186-202

[60] Abys J A 2010 Palladium electroplating Modern Electroplating (Germany: Wiley-VCH Verlag GmbH \& Co. KGaA) pp 327-68

[61] Adams B D and Chen A 2011 The role of palladium in a hydrogen economy Mater. Today 14 282-9

[62] Yang S et al 2015 Tuning the optical, magnetic, and electrical properties of $\mathrm{ReSe}_{2}$ by nanoscale strain engineering Nano Lett.

15 1660-6

[63] Zhu C et al 2019 Strain engineering in perovskite solar cells and its impacts on carrier dynamics Nat. Commun. 10815

[64] Muralidharan N, Carter R, Oakes L, Cohn A P and Pint C L 2016 Strain engineering to modify the electrochemistry of energy storage electrodes $S c i$. Rep. 627542 